\title{
Nonsingular instantons for the creation of open universes
}

\author{
Pedro F. González-Díaz \\ Centro de Física “Miguel Catalán,”, Instituto de Matemáticas y Física Fundamental, Consejo Superior de Investigaciones Científicas, \\ Serrano 121, 28006 Madrid, Spain
}

(Received 4 May 1998; revised manuscript received 18 August 1998; published 21 January 1999)

\begin{abstract}
We show that the instability of the singular Vilenkin instanton describing the creation of an open universe can be avoided using, instead of a minimally coupled scalar field, an axionic massless scalar field which gives rise to the Giddings-Strominger instanton. However, if we replace the singularity of the Hawking-Turok instanton for an axionic wormhole some interpretational and technical difficulties would appear which can be overcome by introducing a positive cosmological constant in the action. This would make the instanton finite and free from any instabilities. [S0556-2821(99)05302-3]

PACS number(s): 98.80.Cq, 04.60.-m
\end{abstract}

\section{INTRODUCTION}

One of the most promising recent developments in theoretical cosmology is the proposal by Hawking and Turok [1] of a singular instanton for the creation of open universes. It has prompted an almost inmediate influx of comments, criticisms, replies, and new work on the subject [2]. The relevance of this proposal resides on the fact that it satisfies the need for formulating open inflationary models with $\Omega<1$ which is required by recent observational estimates of the present value of the Hubble constant [3-6] and the density of gravitational lenses in the universe $[7,8]$. Most available inflationary models predicts a value for $\Omega$ very close to unity and cannot therefore conform these observations.

Hawking and Turok used, as a solution to the Euclidean Einstein equations [1],

$$
d s^{2}=d \sigma^{2}+b(\sigma)^{2}\left(d \psi^{2}+\sin ^{2} \psi d \Omega_{2}^{2}\right),
$$

with $d \Omega_{2}^{2}$ the metric on the unit two-sphere. They continued Eq. (1.1) into the metric for an open spatially homogeneous universe with a real scale factor by first matching Eq. (1.1) to the inhomogeneous de Sitter-like Lorentzian solution

$$
d s^{2}=d \sigma^{2}+b(\sigma)^{2}\left(-d \tau^{2}+\cosh ^{2} \tau d \Omega_{2}^{2}\right),
$$

across the surface $\psi=\pi / 2$, with the continuation $\psi=(\pi / 2)$ $+i \tau$, and then continued Eq. (1.2) into the metric of a Lorentzian isotropic open universe

$$
d s^{2}=-d t^{2}+a(t)^{2}\left(d \chi^{2}+\sinh ^{2} \chi d \Omega_{2}^{2}\right),
$$

with the rotation $\tau=(\pi / 2) i+\chi, \sigma=i t, b(i t)=i a(t)$, at the singular surface $\sigma=0$, where $b=\sigma$.

The main criticism to this singular instantonic model has been raised by Vilenkin [9] who showed that singular instantons of the type suggested by Hawking and Turok, but more manageable and actually able to provide exact solutions as we shall see in this paper, lead to the physically unacceptable consequence that the resulting universe would be overrun by a high density of expanding singular bubbles. In order to avoid this problem, Garriga [10] regularized the instanton singularity with matter, coupling a membrane to the scalar field, and more recently [11], considering a nonsingular five- dimensional instanton whose four-dimensional sections are the Hawking-Turok instanton. In this paper we shall regularize the singularity of the latter instanton by using quite a different procedure: Instead of introducing any new ingredient in the background theory, we will modify the nature of the scalar field itself and/or its coupling to gravity. The result is also a change in the instanton structure. We have seen that in order to avoid the singularity of the Hawking-Turok instanton or the asymptotically flat instanton used by Vilenkin [9], one should replace them for an axionic wormhole which becomes a nonsingular finite instanton if a positive cosmological constant is introduced in the action.

We outline the paper as follows. In Sec. II we review the asymptotically flat instanton introduced by Vilenkin [9] by using a closed form solution for it. Basing on the resulting formalism we analyze in Sec. III the kind of change that we must introduce in the matter field to obtain a nonsingular instanton able to replace the Hawking-Turok instanton, instead of the Vilenkin solution. What results from such a change is the known Giddings-Strominger instanton [12], which we review in terms of the conformal time, and discuss in relation with the creation of an open universe along the lines of the Hawking-Turok model. This analysis is extended in Sec. IV to include a positive cosmological term in the distinct wormhole solutions. Thus, two new candidates, the instantons first considered by Halliwell and Laflamme [13] and by Myers [14], emerge as suitable regularized substitutes of the singularity of the Hawking-Turok instanton. We also review these solutions in terms of the conformal time and consider them in the framework of the instantonic creation of open universes. In Sec. V we consider the global structure of the introduced spacetimes in the context of the HawkingTurok model. We finally conclude in Sec. VI where some comments on the obtained results are added.

\section{THE SINGULAR INSTANTON}

In this section we review the characteristics of the asymptotically flat singular instanton introduced by Vilenkin [9]. The analysis to follow is based on the derivation of a closed form solution for such an instanton. The model is very simple. It corresponds to a massless scalar field $\phi$ which is minimally coupled to Hilbert-Einstein gravity. The Euclid- 
ean action for this model is [9]

$$
S_{E}=\int d^{4} x \sqrt{g}\left[-\frac{R}{16 \pi G}+\frac{1}{2}(\partial \phi)^{2}\right]+S_{\text {boundary }} .
$$

The instantonic solution will be $\mathrm{O}(4)$ symmetric and, when described by metric (1.1), the equations of motion for the scale factor $b(\sigma)$ and massless scalar field $\phi \equiv \phi(\sigma)$ are

$$
\begin{gathered}
\phi^{\prime \prime}+3 \frac{b^{\prime}}{b} \phi^{\prime}=0, \\
b^{\prime \prime}=-\frac{8 \pi G}{3} b \phi^{\prime 2},
\end{gathered}
$$

with the primes standing for derivatives with respect to the Friedmann-Robertson-Walker time $\sigma$. From Eq. (2.2) we have $\phi^{\prime}=-C / b^{3}$, with $C$ a real integration constant. After integration, Eq. (2.3) then becomes

$$
b^{\prime 2}-\frac{l_{p}^{2} C^{2}}{b^{4}}=1
$$

where $l_{p}=\sqrt{4 \pi G / 3}$ and the integration constant has been chosen to be unity in order to satisfy the necessary conditions that correspond to an asymptotically flat solution, i.e., $b(\sigma) \approx \sigma$ and $\phi(\sigma) \rightarrow 0$ as $\sigma \rightarrow \infty$.

We now note that the equation of motion (2.4) admits an exact analytical solution. This can be most easily seen if we introduce the conformal time $\Sigma$, defined by

$$
\Sigma=\int_{0}^{\sigma} \frac{d \sigma^{\prime}}{b\left(\sigma^{\prime}\right)}
$$

so that

$$
b^{2} \dot{b}^{2}-b^{4}-l_{p}^{2} C^{2}=0
$$

with $=d / d \Sigma$. The solution to Eq. (2.6) reads

$$
b(\Sigma)=\sqrt{C l_{p}} \sinh ^{1 / 2}(2 \Sigma) .
$$

From Eq. (2.5) we also obtain

$$
\begin{aligned}
\sigma-\sigma_{*}= & \sqrt{C l_{p}}\left\{\frac{1}{2}[F(\alpha, r)-2 E(\alpha, r)]\right. \\
& \left.+\frac{b(\Sigma) \sqrt{C^{2} l_{p}^{2}+b(\Sigma)^{4}}}{\sqrt{C l_{p}}\left(C l_{p}+b(\Sigma)^{2}\right)}\right\}
\end{aligned}
$$

where $F$ and $E$ are the elliptic integral of the first and second kind, respectively, $\quad \alpha=\arccos \left\{\left[C l_{p}-b(\Sigma)^{2}\right] /\left[C l_{p}\right.\right.$ $\left.\left.+b(\Sigma)^{2}\right]\right\}, r=\sqrt{2} / 2$, and $\sigma_{*}$ is an integration constant. Clearly, for $\Sigma=0, \sigma=\sigma_{*}$, while for $\Sigma \rightarrow \infty, \sigma \rightarrow \infty$.

An analytical solution for the field $\phi$ can also be obtained in terms of the conformal time $\Sigma$. From the conservation of the matter field charge, $b^{2} \dot{\phi}=-C$, one gets

$$
\phi=-\frac{1}{4 l_{p}} \ln \left(\frac{\cosh 2 \Sigma-1}{\cosh 2 \Sigma+1}\right)+\phi_{*} .
$$

In order to satisfy the boundary conditions in the asymptotic region one should set $\phi_{*}=0$. Solutions (2.7) and (2.9) satisfy then all the wanted requirements, i.e., as one moves towards smaller values of $\sigma, b(\sigma)$ decreases and $\phi(\sigma)$ grows until $b$ vanishes and $\phi$ logarithmically diverges at $\sigma$ $=\sigma_{*}$.

As it was already noted in Ref. [9], the only contribution to the Euclidean action comes from the boundary term at $\sigma$ $=\sigma_{*}$, and is given by

$$
S_{E}=S_{\text {boundary }}=-\frac{1}{8 \pi G} \partial_{\text {normal }} V_{B},
$$

(with $V_{B}$ the volume of the boundary) which, using Eq. (2.5), reproduces the value $S_{E}=\sqrt{3 \pi^{2} / 4 G} C$ obtained by Vilenkin [9].

Of course, this instanton is an $\mathrm{O}(4)$ instanton which can be described by the Hawking-Turok metric

$$
d s^{2}=b(\Sigma)^{2}\left(d \Sigma^{2}+d \psi^{2}+\sin ^{2} \psi d \Omega_{2}^{2}\right)
$$

which should first be matched to the spatially inhomogeneous de Sitter-like solution

$$
d s^{2}=b(\Sigma)^{2}\left(d \Sigma^{2}-d \tau^{2}+\cosh ^{2} \tau d \Omega_{2}^{2}\right)
$$

across the surface $\psi=\pi / 2$, by making the continuation $\psi$ $=\pi / 2+i \tau$, and then continued again into the metric of a Lorentzian isotropic open universe

$$
d s^{2}=a(\Sigma)^{2}\left(-d \eta^{2}+d \chi^{2}+\sinh ^{2} \chi d \Omega_{2}^{2}\right)
$$

where $d \eta=d \sigma / a(\Sigma)$, by using the additional continuation $\Sigma \rightarrow-\Sigma$ (i.e., $\sigma=i t), \tau=(\pi / 2) i+\chi$, and $b(-\Sigma)=i a(\Sigma)$, at the singular surface $\sigma=\sigma_{*}$. The singular behavior of this instanton is exactly the same as that of the Hawking-Turok instanton, but has the physically unacceptable property of predicting the creation of an open universe filled with expanding singular bubbles [9].

\section{REPLACING THE SINGULARITY WITH A WORMHOLE}

From the conservation of the matter field charge $(d / d \Sigma)\left(b^{2} \dot{\phi}\right)=0$, one can generally write $b^{2} \dot{\phi}=\mp C$, with $C$ an integration constant. For ordinary fields where $C$ is real, we obtain the singular instanton reviewed in Sec. II. However, if the considered instanton arises from an axionic conserved charge, to examine instantonic transitions with fixed axion charge, we must then modify the Euclidean action (2.1) by an additional surface term which is given by

$$
S_{A}=-\left.b^{2} \phi \dot{\phi}\right|_{0} ^{T},
$$

and hence [14] one obtains from the conservation of the momentum conjugate to $\phi$ that we must choose the constant $C$ 
to be pure imaginary, i.e., $b^{2} \dot{\phi}= \pm i C$, and therefore, instead of Eq. (2.6), we get for the equation of motion for the scale factor

$$
b^{2} \dot{b}^{2}-b^{4}+l_{p}^{2} C^{2}=0
$$

and hence, instead of the singular instanton solution (2.7), (2.9), we have as a closed form solution for the instanton

$$
\begin{aligned}
& b(\Sigma)=\sqrt{C l_{p}} \cosh ^{1 / 2}(2 \Sigma), \\
& \phi(\Sigma)=\frac{i}{2 l_{p}} \arcsin (\tanh 2 \Sigma)+\phi_{*},
\end{aligned}
$$

where in this case $\phi_{*}=-i \pi / 4 l_{p}$, and

$$
\sigma-\sigma_{*}=\sqrt{C l_{p}}\left\{\frac{1}{\sqrt{2}}[F(\alpha, r)-2 E(\alpha, r)]+\frac{\sqrt{C l_{p}} \sinh 2 \Sigma}{b(\Sigma)}\right\},
$$

with $\alpha=\arcsin \left\{\left[b(\Sigma)^{2}-C l_{p}\right] / b(\Sigma)^{2}\right\}$ and $r=\sqrt{2} / 2$. Again, for $\Sigma=0, \sigma=\sigma_{*}$, and for $\Sigma \longrightarrow \infty, \sigma \rightarrow \infty$. However, the imaginary field $\phi$ will now become $\phi_{*}$ at $\Sigma=0$ and vanish asymptotically. The differences between the instantons corresponding to the real scalar field and those associated with pure-imaginary values of the scalar field have been discussed in Ref. [15]. The scale factor (3.3) corresponds to a nonsingular instanton which is nothing but the known GiddingsStrominger wormhole [12]. Using Eq. (2.10) for this instanton, we obtain $S_{\text {boundary }}=0$, which, by taking into account Eq. (3.1), in turns implies that the Euclidean action $S_{E}$ $=(\sqrt{3 \pi / 16 G}) C$. Thus, the nonsingular instanton (3.3), (3.4) appears to be more stable than the singular instanton (2.7), (2.9) and, moreover, would be free from the instabilities Vilenkin pointed out [9] for this.

The new nonsingular instanton will also be able to describe the creation of an open inflationary universe. In fact, for solutions (3.3) and (3.4) one can also continue the metric for the spatially inhomogeneous solution into the metric of a nonsingular Lorentzian isotropic open universe

$$
d s^{2}=a(\Sigma)^{2}\left(-d \Sigma^{2}+d \chi^{2}+\sinh ^{2} \chi d \Omega_{2}^{2}\right)
$$

by continuing Eq. (2.11) so that $C \rightarrow-C, \tau=(\pi / 2) i$ $+\chi, b(\Sigma, C)=i a(\Sigma,-C)$, at the minimal nonsingular surface $\sigma=\sigma_{*}$. Thus, if we replace the singularity of the Hawking-Turok instanton for one of these wormholes, then the instability pointed out by Vilenkin would be avoided. This solution would somewhat be related to that recently suggested by Garriga [10] in terms of a scalar field coupled to a membrane in that both solutions avoid the instability problem by smoothing the singularity out.

Thus, when the Giddings-Strominger wormhole with scale factor (3.3) is used in the Hawking-Turok metric (2.11), it, too, is able to describe creation of an open inflationary universe. This is a novel result which does not contradict the original interpretation given by Giddings and Strominger [12] for Euclidean wormholes, according to which a wormhole instanton describes a microscopic connection between two asymptotically flat regions belonging to the same universe, or to two different universes, but can be thought to complete it whenever one allows the wormhole to also become the seed for the creation of an open universe along the lines discussed above and in more detail in Sec. V. What is actually new here is the use being given to a wormhole when this is continued from the Euclidean region into a spacetime described by metric (3.6), even if we keep the original interpretation for wormholes given by Giddings and Strominger.

Replacing the singularity of the Hawking-Turok instanton for an axionic wormhole leads in turn to other difficulties. First of all, we have an interpretational problem. Since the wormhole is supposed to connect two nearly flat spacetime regions, one would consider what is this wormhole connected to. This problem could be solved by simply connecting the wormhole to another copy of the instanton; this possibility was pointed out to us by Vilenkin [16], but one does not know for sure how it could be implemented physically. On the other hand, one might alternatively adopt another approach to the problem by renouncing the idea that the universe is created from nothing, replacing it for the notion of universe creation from a pre-existing large universe which the wormhole would be connected to.

Another more serious difficulty with the idea of an axionic wormhole smoothing out the singularity is that the Giddings-Strominger instanton tuns out to be instable against fluctuations of the metric. Such a wormhole possesses a negative mode which would induce instabilities of flat space to the creation of baby universes [17].

\section{NONSINGULAR FINITE INSTANTONS}

The wormhole instanton discussed in Sec. III corresponds to a solution of the Euclidean Einstein equations with vanishing cosmological constant and no scalar field potential, $V(\phi)=0$. The asymptotically flat or anti-de Sitter behavior [18] of wormhole spacetimes is lost when a nonzero potential $V(\phi)>0$ and/or a positive cosmological constant $\Lambda>0$ is included in the corresponding action. The resulting instantons are then seen to be nonsingular and connect given surfaces of finite size. If we take them to replace the singularity of the Hawking-Turok instanton, such instantons would not show any of the problems pointed out at the end of the precedent section. Taking advantage of this fact, we shall include a positive cosmological term in the wormhole instantonic action and replace the singularity of the HawkingTurok instanton for half of the resulting nonsingular finite instantons. We will divide the instantons in two equal halves by slicing the space through the minimal surface at the neck, i.e., at the surface of minimal size.

There are two possible such instantons. We first consider the case where a massless scalar field is conformally coupled to Hilbert-Einstein gravity, adding a positive cosmological term. The Euclidean action is then 


$$
\begin{aligned}
S_{E}= & -\frac{1}{16 \pi G} \int d^{4} x \sqrt{g}\left[\left(1-l_{p}^{2} \phi^{2}\right) R-2 \Lambda\right] \\
& +\frac{1}{2} \int d^{4} \sqrt{g}(\nabla \phi)^{2}-\frac{1}{8 \pi G} \int d^{3} x \sqrt{h}\left(1-l_{p}^{2} \phi^{2}\right) \operatorname{Tr} K,
\end{aligned}
$$

with $K$ the second fundamental form.

For the metric (1.1) one obtains then the HalliwellLaflamme nonsingular instanton [13]. We have found the closed form solution in terms of the conformal time $\Sigma$ for this instanton

$$
b(\Sigma)=\frac{\xi+1}{\sqrt{2 \lambda / 3}}\left[1+\xi\left(1-2 s n^{2} \eta\right)\right]^{-1 / 2}
$$

where $s n$ is the Jacobean elliptic function, and we have introduced the following definitions:

$$
\begin{gathered}
\xi=\sqrt{1-4 R_{0} \lambda}, \\
\eta=\sqrt{\frac{\xi+1}{2} \Sigma,},
\end{gathered}
$$

with $R_{0}$ an integration constant and $\lambda=l_{p}^{2} \Lambda / 6 \pi^{2}$. For solution (4.2) $K$ and $\lambda$ are subject to the condition $4 K \lambda<1$. Thus, $b$ will vary between the two extreme values $b(0)$ $=(\xi+1) / \sqrt{2 \lambda(\xi+1) / 3}$ (minimal surface) and $b(K)=(\xi$ $+1) / \sqrt{2 \lambda(1-\xi) / 3}$ (maximal surface), the value $\eta=K$ being the quarter-period of the Jacobean elliptic function $s n \eta$. This instanton can also be used as the solution for the scale factor in the Hawking-Turok metric (2.11) in order to describe creation of open inflationary universes. Metric (2.11) can then be continued first into Eq. (2.12) with the rotation $\psi=\pi / 2+\tau$, without changing the scale factor (4.2), and then into (2.13) by using the transformation of the parameters entering the Halliwell-Laflamme instanton (4.2) $\Sigma \rightarrow \Sigma, \xi$ $\rightarrow \xi, \Lambda \rightarrow-\Lambda, K \rightarrow-K$, which correspond to the time rotation $\sigma \rightarrow$ it and

$$
b(\Sigma,-\Lambda,-K)=i a(\Sigma, \Lambda, K)=i b(\Sigma, \Lambda, K) .
$$

As it was already pointed out by Halliwell and Laflamme [13], this instanton has the problem that the effective gravitational constant can become negative when the field $\phi$ exceeds some fixed value. This problem can be avoided if, instead of a conformally coupled scalar field, we use a minimally coupled axionic field with a positive cosmological constant. The resulting instantons were first considered by Myers [14], though he did not get the solutions in closed form.

In order to check that the axionic finite instantons can be adapted to the Hawking-Turok continuation leading to metric (2.12) for a Lorentzian isotropic open universe, let us obtain a closed form solution for their simplest three-dimensional case whose Euclidean action can be written as

$$
\begin{aligned}
S_{E}= & -\frac{1}{16 \pi G} \int d^{3} x \sqrt{g}(R-2 \Lambda)-\frac{1}{2} \int d^{3} x \sqrt{g}(\nabla \phi)^{2} \\
& -\frac{1}{8 \pi G} \int d^{2} x \sqrt{h} K+S_{A}
\end{aligned}
$$

where $S_{A}$ is an axionic boundary term which, for the three dimensional instantonic metric

$$
d s^{2}=b(\Sigma)^{2}\left(d \Sigma^{2}+d \theta^{2}+\sin ^{2} \theta d \varphi^{2}\right),
$$

can be written as $S_{A}=-\left.b \dot{\phi} \phi\right|_{0} ^{T}$, in the gauge where the lapse function is set to $N=1$. Restricting the Myers instanton to be three-dimensional is here required by the fact that three is the highest possible spacetime dimensionality for this instanton which is able to allow exact solutions of the field equations. However, all the geometric properties of the threedimensional Myers instanton, particularly its capability to describe creation of open inflationary universes, can be readily extrapolated to the physically more interesting fourdimensional case.

From the equations of motion for $\phi$ and $b$, we get

$$
\dot{\phi}=\frac{i m}{b^{2}},
$$

$$
\dot{b}^{2}+m^{2}+\Lambda b^{4}=1
$$

The solution to Eqs. (4.8) and (4.9) can still be given in closed form as

$$
\begin{aligned}
b(\Sigma) & =\frac{1}{\sqrt{2 \Lambda}} \sqrt{1+\zeta\left(1-2 s n^{2} \delta\right)}, \\
\phi & =i \arctan \left[\frac{\tan (\sqrt{\Lambda} \sigma)+\zeta}{2 m \sqrt{\Lambda}}\right]+\phi_{*},
\end{aligned}
$$

where for the relation between $\sigma$ and $\Sigma$ we have

$$
\sigma-\sigma_{*}=-\frac{1}{2 \sqrt{\Lambda}} \arcsin \left(\frac{1-2 \Lambda b^{2}}{\zeta}\right),
$$

and we have introduced the parameters

$$
\begin{aligned}
& \zeta=\sqrt{1-4 m^{2} \Lambda}, \\
& \delta=\sqrt{\frac{1+\zeta}{2} \Sigma} .
\end{aligned}
$$

We can see from Eq. (4.10) that the scale factor oscillates now between the extreme values $b(0)=\sqrt{(1+\zeta) / 2 \Lambda}$ (maximal surface) and $b(K)=\sqrt{(1-\zeta) / 2 \Lambda}$ (minimal surface), the value $\delta=K$ again being the quarter period of the Jacobean elliptic function $s n \delta$. The Euclidean action for this instanton can be easily computed [14]. It is given by 


$$
S_{E}=\frac{\pi}{2 G}\left(m-\frac{1}{2 \sqrt{\Lambda}}\right)
$$

which is finite and negative. Thus, this instanton will contribute the Euclidean path integral for the creation of open universes.

If we now match the Euclidean metric (4.7) to the threedimensional spatially inhomogeneous solution

$$
d s^{2}=b(\Sigma)\left(d \Sigma^{2}-d \tau^{2}+\cosh ^{2} \tau d \varphi^{2}\right),
$$

across the surface $\theta=\pi / 2$, by making the continuation $\theta$ $=\pi / 2+i \tau$, then, given the form of the scale factor (4.10), the resulting metric $(4.15)$ can be continued into the metric of a Lorentzian isotropic open universe in three dimensions:

$$
d s^{2}=a(\Sigma)\left(-d \Sigma^{2}+d \chi^{2}+\sinh ^{2} \chi d \varphi^{2}\right),
$$

by using the additional continuation $\sigma \rightarrow i t, \Lambda \rightarrow-\Lambda, m$ $\rightarrow i m, \tau \rightarrow(\pi / 2) i+\chi$, so that $\Sigma \rightarrow \Sigma$ and

$$
b(\Sigma, \Lambda, m) \rightarrow i a(\Sigma,-\Lambda, i m)=i b(\Sigma, \Lambda, m) .
$$

Thus, we obtain the wanted Lorentzian isotropic metric for a three-dimensional open universe, provided we take a negative cosmological constant and an ordinary massless nonaxionic field. We note, moreover, that starting with action (4.6), which we perturb introducing $\mathrm{O}(3)$-symmetric fluctuations about the classical instanton solution, $b=b_{\mathrm{cl}}$ $+r$, and evaluate in the quadratic part, one can follow the semiclassical Rubakov-Shvedov procedure [17] to finally obtain a frequency squared for the fluctuation mode given by

$$
\omega^{2} \propto-\Lambda\left(1+\frac{m^{2}}{\Lambda b_{\mathrm{cl}}^{4}}\right) .
$$

So, even at the maximal surface of the instanton, $\omega^{2}$ will always be definite positive when we simultaneously rotate $\Lambda \rightarrow-\Lambda$ and $m \rightarrow i m$. Therefore, the nucleation of this instanton will not induce any instabilities in the resulting Lorentzian open universe.

Although we have not succeeded in obtaining a closed form solution for the corresponding four-dimensional case, it is not difficult to convince oneself that the four-dimensional Myers instanton shares the same general behavior as that we have discussed for Eqs. (4.10), (4.11) and, therefore, it can be used as a suitable substitute for the singularity of the Hawking-Turok instanton for the creation of an open universe from nothing.

\section{GLOBAL STRUCTURE OF WORMHOLE-HAWKING- TUROK SPACETIMES}

We have seen that asymptotically flat Euclidean wormholes and the instantonic tunnelings that result from introducing a positive cosmological constant in the wormhole action can all be used to describe the evolution of the scale factor that enters the Hawking-Turok metric, and hence are able to represent creation of an open inflationary universe by means of a regular instanton. In what follows, we shall dis- cuss the geometrical significance of this result. First of all, since in the coordinates $\sigma, \tau$ of metric (1.2) the surface $\sigma$ $=0$ is a null surface, one can redefine the second continuation leading to metric (1.3) by extending the coordinates beyond the surface $\sigma=0$ which is singular in the HawkingTurok geometry. Then, by using the conformal time $\Sigma$, defined in Eq. (2.5), one can introduce Kruskal-like coordinates [2]

$$
U=e^{-\tau+\Sigma}, \quad V=-e^{\tau+\Sigma}
$$

so that metric (1.2) and its conformal-time equivalent (2.12) can both be rewritten as

$$
d s^{2}=b^{2} e^{-2 \Sigma}\left(-d U d V+\frac{1}{4}(U-V)^{2} d \Omega_{2}^{2}\right)
$$

where

$$
e^{-2 \Sigma}=-\frac{1}{U V}
$$

In order for continuing Eq. (5.2) into the metric of an open spatially homogeneous universe with a real positive definite scale factor and real coordinates for the instantons considered in the present paper and also the Hawking-Turok instanton, we first introduce the new coordinates $T, \chi[2]$ through

$$
U=e^{T-\chi}, \quad V=e^{T+\chi}
$$

and then rotate $\sigma$ and $b$ so that $\sigma=i t, b(i t)=i a(t)$, while keeping coordinate $\tau$ real and unchanged. Hence we get the required metric for an open spatially homogeneous universe

$$
d s^{2}=a^{2}\left(-d T^{2}+d \chi^{2}+\sinh ^{2} \chi d \Omega_{2}^{2}\right) .
$$

The global structure of our wormhole-derived spacetimes can now be investigated by considering the $U, V$ diagram given in Fig. 1. It corresponds to the simplest $V(\phi)=0$ version of a Hawking-Turok instanton in which the singularity has been replaced for either the throat of an asymptotically flat wormhole, or for an extremal surface of the finite nonsingular instantons dealt with in Sec. IV. In the sector defined by coordinates $T, \chi$ on such a diagram the dotted line at $U$ $=V$ will correspond to $\chi=0$, that is the origin of the spherically symmetric $\chi, \theta, \varphi$ coordinate system. For all the cases being considered the dashed line at $U V=-1$, that is at $\sigma$ $=\sigma_{*}$ on the $\tau, \Sigma$ region, which is separated from the region described by coordinates $T, \chi$ along the axis $V=0$, is either the throat of the wormhole (that is to say, the maximal surface of the corresponding baby universe in Lorentzian time), or one of the extremal surfaces (maximal for the axionic instanton and minimal for the Halliwell-Laflamme instanton) of the considered two finite instantons. On that line the field $\phi$ always takes on finite values, both for the axionic and the conformally-coupled field cases. This is the crucial point that distinguishes the spacetime structure of the instantons repre- 


\section{LORENTZIAN}

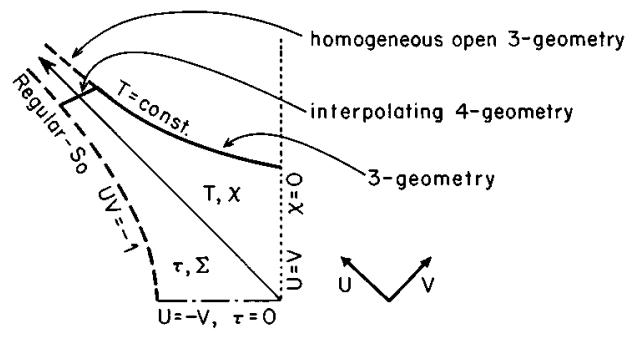

EUCLIDEAN

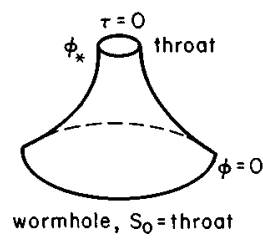

a

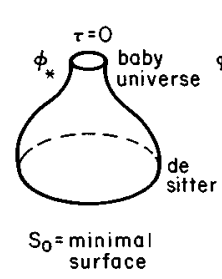

b

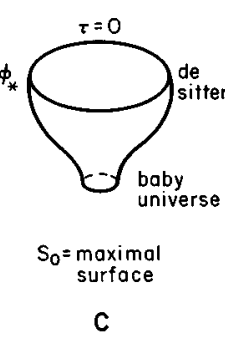

C

FIG. 1. Global structure for the nonsingular instantons. The Euclidean region continues into the Lorentzian one on the surface $U$ $=-V, \tau=0, \phi=\phi_{*}$. It corresponds to (a) an asymptotically flat wormhole instanton, (b) a finite Halliwell-Laflamme instanton, and (c) a finite Myers instanton.

sented in Fig. 1 from that of the Hawking-Turok and Vilenkin instantons for which $\phi$ would diverge on the surface $S_{0}$ (Fig. 1).

Since near the maximal surface of the finite nonsingular instantons the cosmological constant dominates the curvature, that surface corresponds to a de Sitter space deformed by the residual effect of the axion or conformal field, so allowing the resulting instanton to have $\mathrm{O}(4)$ symmetry. Near the minimal surface, it is respectively the axion and the conformal field which dominates the curvature, making a deformed baby universe. It follows that what essentially distinguishes the instantons considered in this paper from the Vilenkin and Hawking-Turok instantons is the presence in the former instantons of a baby universe which (see Fig. 1) (a) replaces the deformed de Sitter space of the HawkingTurok solution (for $\Lambda=0$ ), (b) intercalates after this space (for $\Lambda>0$ and conformally coupled field), and (c) is an initial boundary from which the system tunnels into the deformed de Sitter space (for $\Lambda>0$ and axionic field).

The region $\tau, \Sigma$ can be connected to the Euclidean sector on the surface defined by $\tau=0, \phi=\phi_{*}, U=-V$. The wormhole [case (a)] develops from that surface approaching an asymptotically flat region where $\phi=0$, and the finite instantons do approaching either the maximal finite surface [case (b)] or the minimal surface [case (c)]. In the latter two cases there exists the interesting possibility of sewing together two copies of the whole solution illustrated in Fig. 1 on the Euclidean sector, to give rise to an overall process which can be interpreted as the creation of two open inflationary universes from either tunneling between two baby universes through a deformed de Sitter universe [case (b)] or tunneling between two deformed de Sitter universes through a baby universe when the Myers instanton is considered.

On the other hand, we always can have a four-geometry

on the Lorentzian sector which can be regarded as a valid interpolating geometry (see Fig. 1) between one of the spatial hypersurfaces in the $T, \chi$ region and either the wormhole throat, or the maximal (axionic field) or minimal (conformal field) surface at $\tau<0$. Unlike in the original Hawking-Turok spacetime, in any of the considered cases, any of the threegeometries on $T, \chi$, together with the interpolating fourgeometry and the three-geometry at $\Sigma=0$, is a regular geometry. Note that if the surfaces at $\Sigma=0$ would be replaced by a singularity, then this singularity would engulf the whole spacetime, just as the singularity of the Vilenkin model does [9].

In the language of the prescription for the wave function of the universe, the interpolating four-geometry may be used to calculate the probability for the propagation from, respectively, the wormhole throat or the extremal surfaces of finite instantons to the given three-geometry on the region with $T, \xi$ coordinates. Such a calculation would be performed by looking at the imaginary part of the corresponding action.

Finally, let us briefly comment on the four-dimensional axionic finite instanton within the context of the HawkingTurok procedure, since this instanton appears to be of most physical interest. Although the four-dimensional Myers solution is not exactly solvable in terms of the scale factor in a Robertson-Walker metric, according to Myers [14] one can still write the spherically symmetric metric for such a solution in the form

$$
d s^{2}=\left(1-\frac{\Lambda r^{2}}{3}-\frac{m^{2}}{r^{4}}\right)^{-1} d r^{2}+r^{2} d \Omega_{3}^{2},
$$

where $d \Omega_{3}^{2}$ is the metric on the unit three-sphere and $m$ is an integration constant. Extreme surfaces occur at $g_{r r}=\infty$, which correspond to coordinate singularities as the components of the curvature tensor become all finite there. Then, similarly to as it happens in the three-dimensional case, the radial coordinate, and actually the scale factor, must vary between the extreme values defined by the apparent singularities at $g_{r r}^{-1}=0$. Specializing, e.g., to the particular simplest case where $\frac{3}{4} m^{2} \Lambda^{2}=1$, the extreme values become

$$
r_{ \pm}=\Lambda^{-1} \pm\left(6 m^{3} / \Lambda\right)^{1 / 3} \text {. }
$$

Moreover, a scale factor with extreme values as given by Eq. (5.6) (or by more complicated expressions for more general cases) can also be used in the procedure leading from Eq. (1.1) to Eq. (1.3). Thus, the four-dimensional Myers instanton can be considered as a rather natural ingredient of the global spacetime structure [for Euclidean sector (c) of Fig. 1] of the nonsingular solutions described in this section, and be therefore used as a convenient substitute for the singularity of the Hawking-Turok instanton.

\section{CONCLUSIONS}

Wormholes are genuine components of the quantum spacetime foam. So far, they have been used for a variety of purposes, including as spacetime channels to transfer the information that fell in a black hole into a different universe, to 
induce loss of quantum coherence in ordinary matter at low energy, or most popularly, to implement a mechanism for determining the coupling constants $[12,19]$ and the vanishing of the cosmological constant [20]. In this paper wormholes and their finite instantonic generalizations with a positive cosmological constant are added the new use of playing the role of the instantons required by the mechanism suggested by Hawking and Turok for the creation of open universes [1]. The proposal is based on the realization that the singular asymptotically flat instanton discovered by Vilenkin [9] is nothing but the ordinary scalar field counter part of the axionic Giddings-Strominger instanton [12] that corresponds to a different formulation of the problem.

However, if we choose the nonsingular axionic wormhole to replace the singularity of the Hawking-Turok instanton, new difficulties arise which are of both interpretational and technical nature. In order to overcome these difficulties one should introduce either a nonzero generic potential for the scalar field or a nonvanishing positive cosmological constant, both making the instanton finite. We have considered the solutions that result in the latter case for scalar fields which are both conformally [13] and minimally [14] coupled to gravity, suggesting these solutions as suitable candidates to replace the singularity of the Hawking-Turok instanton for the creation of an open universe. When included in the continuation procedure that gives rise to open universes, the instantons arising from minimally coupling an axionic massless scalar field to gravity in the presence of a positive cosmological constant appear to be free from any of the shortcomings that could lead to interpretational or technical difficulties, within the considered model for the creation of open universes. It appears therefore that the most likely process for the creation of single or paired open universes would include tunneling from the baby universes which are thought [21] to pervade the quantum structure of the vacuum spacetime foam.

Our conclusions are based on the analysis of the different solutions in closed form. However, in order for the creation model to generate an inflationary process and an acceptable value of the cosmological parameter $\Omega<1$ as well, one ought to consider the path integral for Einstein gravity coupled to a scalar field with a nonvanishing matter potential whose shape will ensure $\mathrm{O}(4)$ invariance, which does not allow having classical solutions in closed form. Therefore, acceptable predictions for $\Omega$ in the framework of an appropriate open inflationary model could only be achieved if we include a simple generic potential $V(\phi)$ with just a true minimum at $V=0$ in our wormhole-like model, analyzing all allowed predictions in accordance with procedures similar to those used by Hawking and Turok [1,2].

\section{ACKNOWLEDGMENTS}

For useful correspondence the author thanks A. Vilenkin. This reasearch was supported by DCICYT under Research Project No. PB94-0107.
[1] S.W. Hawking and N. Turok, Phys. Lett. B 425, 25 (1998).

[2] A.D. Linde, Phys. Rev. D 58, 083514 (1998); S.W. Hawking and N. Turok, "Comment on Quantum Creation of an Open Universe, by Andrei Linde," gr-qc/9802062; Z.C. Wu, “Creation of Closed and Open Universe from Constrained Instanton," hep-th/9803121; W. Unruh, "On the Hawking Turok Solution to the Open Universe Wave Function,', gr-qc/9803050; N. Turok and S.W. Hawking, Phys. Lett. B 432, 271 (1998); P.F. González-Díaz, "On the geometry of the Hawking Turok Instanton,' hep-th/9803178; R. Bousso and A.D. Linde, Phys. Rev. D 58, 083503 (1998).

[3] A.G. Riess, W.H. Prees, and R.P. Kirshner, Astrophys. J. Lett. 438, 17 (1995).

[4] J. Mould and W. Freedman, as reported by R. C. Kennicutt Jr., Nature (London) 381, 555 (1996).

[5] A. Sandage et al., as reported by R.C. Kennicutt Jr., Nature (London) 381, 555 (1996).

[6] T. Kundic et al., Astrophys. J. 482, 75 (1997).

[7] E.L. Turner, Astrophys. J. Lett. 365, 43 (1990).

[8] M. Fukugita, T. Futamase, and M. Kasai, Mon. Not. R. Astron. Soc. 246, 24P (1990).

[9] A. Vilenkin, Phys. Rev. D 57, R7069 (1998).

[10] J. Garriga, "Open Inflation and the Singular Boundary,' hep-th/9803210.

[11] J. Garriga, "Smooth Creation of an Open Universe in Five Dimensions,"' hep-th/9804106.

[12] S. Giddings and A. Strominger, Nucl. Phys. B307, 854 (1988); B306, 890 (1988).
[13] J.J. Halliwell and R. Laflamme, Class. Quantum Grav. 6, 1839 (1989).

[14] R.C. Myers, Phys. Rev. D 38, 1327 (1988); Nucl. Phys. B323, 225 (1989).

[15] K. Lee, Phys. Rev. Lett. 61, 263 (1988); S. Coleman and K. Lee, Nucl. Phys. B329, 387 (1990); L.F. Abbott and M.B. Wise, ibid. B325, 687 (1989); O.Yu. Shvedov, in "Proceedings of the 24th ITEP Winter School of Physics," gr-qc/9603039; V.A. Rubakov and O.Yu. Shvedov, in "Proceedings of the International Seminar Quarks-96,' Yaroslavl, Russia, 1996, Vol. 1, p. 206, gr-qc/9608065.

[16] A. Vilenkin (personal communication).

[17] V.A. Rubakov and O.Yu. Shvedov, Phys. Lett. B 383, 258 (1996).

[18] C. Barceló, L.J. Garay, P.F. González-Díaz, and G.A. Mena Marugán, Phys. Rev. D 53, 3162 (1996).

[19] S. Coleman, Nucl. Phys. B307, 867 (1988); G.V. Lavrelashvili, V.A. Rubakov, and P.G. Tinyakov, JETP Lett. 46, 167 (1987); in Quantum Gravity, Proceedings of the 4th International Seminar, Moscow, 1987, edited by M.A. Markov, V.A. Berezin, and V.P. Frolov (World Scientific, Singapore, 1988).

[20] S.W. Hawking, Mod. Phys. Lett. A 5, 145 (1990); 5, 453 (1990); S. Giddings and A. Strominger, Nucl. Phys. B321, 481 (1989).

[21] L.J. Garay, Phys. Rev. Lett. 80, 2508 (1998). 